\title{
Reduced carotid atherosclerosis in asthmatic patients treated with inhaled corticosteroids
}

\author{
M. Otsuki*, A. Miyatake ${ }^{\#}$, K. Fujita ${ }^{\top}$, T. Hamasaki ${ }^{+}$and S. Kasayama*
}

ABSTRACT: Although inflammation is an important component of atherosclerosis, it is unknown whether inhaled corticosteroids (ICS) as anti-inflammatory drugs prevent atherosclerosis.

In the present study, carotid atherosclerosis was evaluated by ultrasonography in 150 asthmatic patients who had been regularly treated with ICS, and in 150 matched nonasthmatic controls, with an assessment of atherosclerotic risk factors.

Carotid intima-media thickness was significantly lower in the asthmatic patients than in the controls. The prevalence of carotid plaque tended to be lower in the asthmatic patients than in the controls. Defined carotid atherosclerosis was diagnosed in $\mathbf{5 1}$ of the asthmatic patients, who were older, with a higher prevalence of males, a higher prevalence of dyslipidaemia and a lower mean daily dose of ICS than the 99 patients without carotid atherosclerosis. Stepwise multiple logistic regression analysis identified age, male sex and dyslipidaemia as positive risk factors for carotid atherosclerosis. The mean daily dose of ICS was a negative risk factor.

Carotid atherosclerosis is reduced in asthmatic patients treated with ICS compared with matched controls. This study suggests that ICS may have protective effects against atherosclerosis.

KEYWORDS: Asthma, atherosclerosis, glucocorticoid receptor, inflammation, inhaled corticosteroid

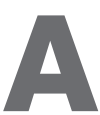
sthma is considered a chronic inflammatory disorder of the airways involving many cells and cellular elements [1]. Inhaled corticosteroids (ICS) are the most effective long-term controller therapy for the treatment of asthma because they have a potent anti-inflammatory effect on the airways [2]. Compared with orally or parenterally administered corticosteroids, ICS are thought to have little effect on tissues other than the airways. However, there is growing concern about systemic absorption and possible adverse systemic effects of ICS [3], which may include suppression of the pituitary-adrenal axis, reduced bone density, increased fracture risk, disturbed growth in children, and cataracts $[4,5]$. Most of these effects have been shown to be dependent on ICS dosage. It has therefore been recommended that the lowest possible ICS dose should be used that is still effective for the maintenance of therapeutic efficacy [6].

It is generally accepted that inflammation plays an important role in the aetiology of atherosclerosis

For editorial comments see page 466 .
[7]. However, administration of corticosteroids to prevent inflammation has the potential to induce or exacerbate several known coronary risk factors, including hypertension, hypercholesterolaemia, hypertriglyceridaemia and impairment of glucose tolerance [8]. A large cohort study by VARASLORENZO et al. [9] showed that the use of oral corticosteroids was associated with a greater risk of acute myocardial infarction in $>40,000$ subjects from the general UK population. Nevertheless, the anti-inflammatory properties of corticosteroids may also exert beneficial effects on the initiation and progression of atherosclerosis. Corticosteroids reportedly have a direct inhibitory effect on the expression of adhesion molecules in vascular endothelial cells, such as intercellular adhesion molecule-1, E-selectin [10] and vascular cell adhesion molecule-1 [11], all of which are thought to be involved in the early process of atherosclerosis [12]. Although the concentrations of ICS entering into the circulation are very low, they may have the potential to inhibit atherosclerosis-related inflammation since this type of inflammation is

\section{AFFILIATIONS}

*Dept of Medicine,

+Dept of Biomedical Statistics, Osaka University Graduate School of Medicine,

\#Miyatake Asthma Clinic, Osaka, and "School of Human Nursing, University of Shiga Prefecture, Shiga Japan.

\section{CORRESPONDENCE}

M. Otsuki

Dept of Medicine

Osaka University Graduate School of Medicine

2-2 Yamada-oka

Suita

Osaka 565-0871

Japan

E-mail: otsuki@

imed3.med.osaka-u.ac.jp

Received:

June 072009

Accepted after revision:

March 102010

First published online:

April 222010 
chronic and moderate. The study by VARAS-LORENZO et al. [9] showed that there was no increase in the risk of acute myocardial infarction associated with the current use of ICS. In another study, it was suggested that ICS administration may be associated with a reduced risk of acute myocardial infarction in asthmatic patients [13]. Based on these observations, we hypothesised that ICS would exert anti-inflammatory effects on the vascular walls and thereby protect against atherosclerosis. In order to examine this, we compared atherosclerosis of the carotid arteries, which has been shown to be directly associated with cardiovascular diseases [14], in asthmatic patients regularly treated with ICS and matched control subjects, and analysed the risk factors for carotid atherosclerosis in the asthmatic patients.

\section{PATIENTS AND METHODS}

\section{Study subjects}

For the evaluation of carotid atherosclerosis, 299 patients were randomly selected from non-hospitalised Japanese asthmatic patients between January 2004 and October 2009 at the Miyatake Asthma Clinic (Osaka, Japan), in accordance with the criteria of the American Thoracic Society (ATS) [15]. All patients had reversible airway obstruction, defined as an increase in forced expiratory volume in $1 \mathrm{~s}$ (FEV1) of $>12 \%$ after short-acting $\beta_{2}$-agonist (salbutamol $0.4-0.8 \mathrm{mg}$ ) inhalation. Of the 299 patients, those meeting the following inclusion criteria were enrolled in the study: $<65$ yrs old, no regular use of oral corticosteroids and/or intravenous corticosteroids, and regular use of ICS for the preceding $\geqslant 2$ yrs. There were 150 patients (76 males) who met the inclusion criteria (fig. 1). Their mean \pm SD age was $55.2 \pm 7.6$ yrs and duration of asthma was $18 \pm 14$ yrs (table 1). Forced vital capacity (FVC), expressed as $\%$ predicted, was $86.9 \pm 17.0 \%$ and the $\mathrm{FEV} 1 / \mathrm{FVC}$ ratio was $73.6 \pm 9.2 \%$ before bronchodilator treatment. Blood eosinophil counts were $328 \pm 292 \cdot \mu \mathrm{L}^{-1}$, while the median (25th-75th percentile) serum immunoglobulin (Ig)E concentrations were $260 \mathrm{IU} \cdot \mathrm{mL}^{-1}$ (91-593). During the preceding $2 \mathrm{yrs}, 83(55.3 \%)$ patients had used oral corticosteroids and/or ICS for the treatment of an acute exacerbation of asthma and their average equipotent dose of prednisolone was $3.9 \pm 3.6 \mathrm{mg} \cdot \mathrm{month}^{-1}$; there were no remarkable events, such as requiring emergency treatment or hospitalisation. Some patients were concomitantly treated with inhaled short- and/or long-acting $\beta_{2}$-agonist, inhaled disodium cromoglycate, theophylline and/or leukotriene receptor antagonist when necessary.

All the patients underwent complete physical and laboratory examinations. FVC and FEV1 were measured spirometrically (Autospiro AS-600; Minato Medical Science, Osaka, Japan). A diagnosis of hypertension was based on either a systolic blood pressure of $\geqslant 140 \mathrm{mmHg}$ or a diastolic blood pressure of $\geqslant 90 \mathrm{mmHg}$ by at least two measurements in the sitting position or the use of at least one antihypertensive drug. A diagnosis of dyslipidaemia was based on either a serum low-density lipoprotein cholesterol level of $\geqslant 3.60 \mathrm{mmol} \cdot \mathrm{L}^{-1}$, a serum triglyceride level of $\geqslant 4.61 \mathrm{mmol} \cdot \mathrm{L}^{-1}$, a serum high-density lipoprotein cholesterol level of $<1.03 \mathrm{mmol} \cdot \mathrm{L}^{-1}$, or administration of at least one hyperlipidaemic drug. Diabetes mellitus was diagnosed using the American Diabetes Association criteria [16]. Smoking status was classified on the basis of interview questions and as never smoker, former smoker or current smoker. Patients with asthma were individually matched to control subjects selected from nonasthmatic patients at the Osaka University Hospital (Osaka, Japan) on the basis of age, sex and other atherosclerotic risk factors (diabetes mellitus, hypertension, dyslipidaemia and smoking status). There were 150 matched control subjects (76 males and 74 females) who met the inclusion criteria; their mean age was $54.9 \pm 8.3$ yrs (table 1).

The institutional ethics committee of the Miyatake Asthma Clinic (Osaka, Japan) approved the protocols for this study and all participants gave verbal informed consent.

\section{ICS}

A mean daily dose of ICS was calculated from cumulative doses of ICS administered during the preceding 2 yrs. Of the 150 patients with asthma, during the study period, 63 were treated with inhaled fluticasone propionate (FP; Flutide; GlaxoSmithKline, Tokyo, Japan), 63 with inhaled budesonide (BUD; Pulmicort; AstraZeneca, Osaka, Japan), eight with inhaled hydrofluoroalkane beclomethasone dipropionate (BDP; Qvar; Schering-Plough, Tokyo, Japan), eight with inhaled salmeterol/ FP (Seretide; GlaxoSmithKline), six with inhaled hydrofluoroalkane ciclesonide (CIC) (Alvesco; Teijin Pharma, Tokyo, Japan) and two with inhaled chlorofluorocarbon BDP (Aldecin; Schering-Plough). The daily dose of ICS was adjusted to an equipotent dose: with the dose of chlorofluorocarbon BDP as reference, the conversion factors used to calculate equipotent doses were 1.25 for BUD and 2.0 for FP, hydrofluoroalkane BDP and hydrofluoroalkane CIC [17-19].

\section{Carotid atherosclerosis}

To evaluate atherosclerosis of the carotid arteries, highresolution ultrasound B-mode imaging was performed with an echotomographic system (LOGIQ 200 MD; GE Yokogawa Medical Systems, Tokyo, Japan) using a 9.0-MHz transducer. The ultrasonographic measurement was manual and performed by the same operator who was unaware of the clinical status of the study subjects. Scanning of the extracranial carotid arteries in the neck was performed bilaterally in three different longitudinal projections (anterior, lateral and posterioroblique), as well as the transverse projection [20]. The intima and media thickness (IMT) of the carotid arteries (the distance between the lumen-intima interface and the media-adventitia interface) was measured at three different sites: the greatest thickness (max IMT) and two other points, $1 \mathrm{~cm}$ upstream and $1 \mathrm{~cm}$ downstream from the site of the greatest thickness. The mean of the three measurements of the right and left IMT was defined as the mean IMT, as described previously [21]. A plaque lesion was defined as a distinct area with an IMT 50\% greater than that of neighbouring sites [22]. Carotid atherosclerosis was defined as mean IMT $\geqslant 1.1 \mathrm{~mm}$ and/or the presence of a plaque lesion [21]. The intra-individual coefficient variations of max IMT and mean IMT were $5.5 \%$ and $3.9 \%$, respectively (for eight healthy subjects aged 35.4 \pm 9.7 yrs measured twice at intervals of $3.1 \pm 0.8$ days).

\section{Analysis of data}

We used all computerised profiles of asthmatic patients and control subjects, blinded to the ICS dose and atherosclerosis, after removing patient identifiers. The results are given as mean \pm SD or median (interquartile range). Subject characteristics 


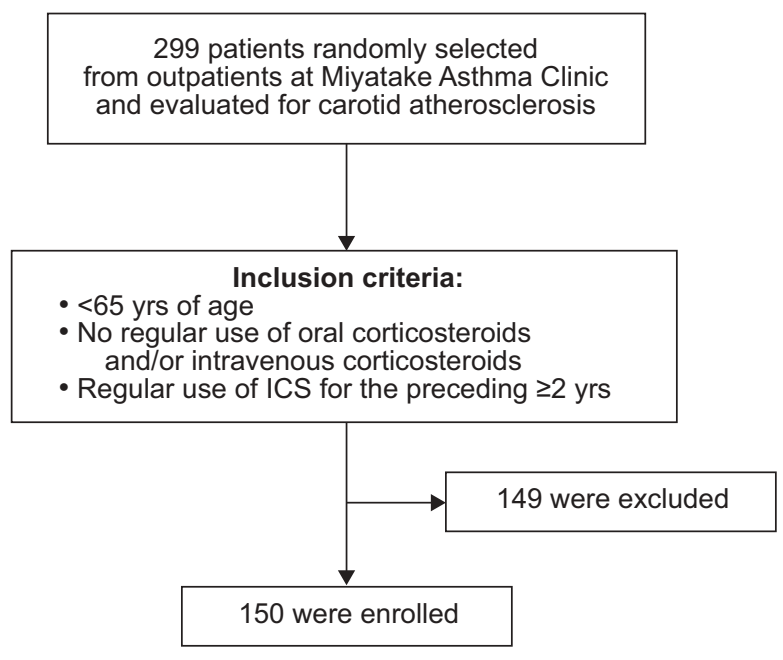

FIGURE 1. Enrolment of asthmatic patients in the study. ICS: inhaled corticosteroids.

for the two groups were compared using an unpaired t-test for continuous variables and a Chi-squared test for categorical variables. To investigate the risk factors for carotid atherosclerosis, stepwise multiple and simple logistic regression analyses were applied with 11 variables (age, male sex, body mass index (BMI), hypertension, dyslipidaemia, diabetes mellitus, smoking status, mean daily dose of ICS, duration of asthma, FVC \% pred and FEV1/FVC \%). The statistical analyses were performed using SAS version 9.1 for Windows (SAS Institute, Cary, NC, USA). The Akaike information criterion (AIC) [23], which is a measure of the goodness of fit of the estimated models, was used for variable selection in this stepwise multiple logistic regression analysis. The stepwise multiple logistic regression analysis results are reported as odds ratios with $95 \%$ confidence intervals. Two-sided p-values of $<0.05$ were considered statistically significant.

\section{RESULTS}

Table 1 shows the demographic characteristics of the asthmatic patients and the control subjects. There were no significant differences in age, sex, BMI and the prevalence of hypertension, dyslipidaemia and diabetes mellitus between the two groups. The prevalence of smoking status was significantly higher in the asthmatic patients than in the control subjects $(p=0.04)$. The mean IMT and max IMT of the carotid arteries of the asthmatic patients was significantly less than that of the control subjects $(p=0.002$ and $p<0.0001$, respectively). The prevalence of carotid plaque tended to be lower in the asthmatic patients than in the control subjects, although the difference did not reach statistical significance $(p=0.06)$. The prevalence of atherosclerotic change in the carotid arteries was significantly lower in the asthmatic patients than in the control subjects $(p=0.03)$.

Table 2 shows the characteristics of the asthmatic patients according to the presence or absence of carotid atherosclerosis, based on the definition given previously. Carotid atherosclerosis was diagnosed in 51 of the 150 asthmatic patients. The patients with atherosclerosis were older $(p=0.008)$, and a higher prevalence of males $(p=0.013)$ and of dyslipidaemia $(p=0.003)$ was seen than in those without carotid atherosclerosis. There was no significant difference in BMI, smoking status or the prevalence of

\begin{tabular}{|c|c|c|c|}
\hline $\begin{array}{l}\text { TABLE } 1 \text { Charact } \\
\text { matchec }\end{array}$ & $\begin{array}{l}\text { s of asthn } \\
\text { trol subjec }\end{array}$ & patients & \\
\hline Characteristic & Patients & Controls & $p$-value \\
\hline Subjects n & 150 & 150 & \\
\hline Males & $76(50.7)$ & $76(50.7)$ & 1.0 \\
\hline Age yrs & $55.2 \pm 7.6$ & $54.9 \pm 8.3$ & 0.70 \\
\hline BMI $\mathrm{kg} \cdot \mathrm{m}^{-2}$ & $24.4 \pm 3.8$ & $23.9 \pm 3.3$ & 0.25 \\
\hline Hypertension & 59 (39.3) & $68(45.3)$ & 0.29 \\
\hline Dyslipidaemia & $81(54.0)$ & $79(52.7)$ & 0.82 \\
\hline Diabetes mellitus & $14(9.3)$ & $15(10.0)$ & 0.85 \\
\hline \multicolumn{4}{|l|}{ Smoking status } \\
\hline Never smoker & $76(50.7)$ & $94(62.7)$ & 0.04 \\
\hline Former smoker & $49(32.7)$ & $30(20.0)$ & \\
\hline Current smoker & $25(16.6)$ & $26(17.3)$ & \\
\hline Mean IMT mm & $0.93 \pm 0.18$ & $1.01 \pm 0.25$ & 0.002 \\
\hline Max IMT mm & $1.02 \pm 0.22$ & $1.18 \pm 0.34$ & $<0.0001$ \\
\hline Plaque & $30(20.0)$ & $44(29.3)$ & 0.06 \\
\hline Carotid atherosclerosis & $51(34.0)$ & $69(46.0)$ & 0.03 \\
\hline
\end{tabular}

hypertension and diabetes mellitus between patients with and without atherosclerosis. Respiratory function expressed as FVC $\%$ pred and FEV1/FVC \% showed no major difference between the two groups. Interestingly, the mean daily dose of ICS was significantly lower for patients with carotid atherosclerosis than for those without $(p=0.02)$. There was no significant difference between patients with and without atherosclerosis in the proportion of patients treated with inhaled $\beta_{2}$-agonists (43.1 versus $44.4 \% ; \mathrm{p}=0.88)$, inhaled disodium cromoglycate $(80.4$ versus $70.7 \% ; \mathrm{p}=0.19)$, theophylline (13.7 versus $19.2 \% ; \mathrm{p}=0.40$ ) and leukotriene receptor antagonists (6.1 versus $7.8 \%$; $=0.68)$.

Table 3 shows the simple logistic regression and stepwise multiple logistic regression analyses in asthmatic patients. Among various factors, dyslipidaemia $(p=0.004)$, male sex $(p=0.015)$ and age $(p=0.026)$ were identified as positive risk factors for carotid atherosclerosis, whereas the mean daily dose of ICS ( $p=0.023$ ) was found to be a negative risk factor. BMI, hypertension, diabetes mellitus, smoking status, duration of asthma, FVC \% pred and FEV1/FVC \% were not significant contributory risk factors for atherosclerosis. In a stepwise multiple logistic regression analysis, male sex $(p<0.001)$, age $(p=0.001)$ and dyslipidaemia $(p=0.018)$ were significantly related to atherosclerosis, while the mean daily dose of ICS $(p=0.021)$ was a significant negative risk factor.

\section{DISCUSSION}

Corticosteroids have metabolic effects that could induce or exacerbate hypertension, hyperglycaemia, hypercholesterolaemia and hypertriglyceridaemia, all of which could be proatherogenic [8]. It has therefore been assumed that corticosteroid use promotes atherosclerosis, although this important issue is not yet settled [24]. However, since atherosclerosis has now been established as an inflammatory disease [7], the anti-inflammatory properties of corticosteroid drugs may be able to mitigate atherosclerotic vascular diseases. 
TABLE 2 Characteristics of asthmatic patients according to the presence or absence of carotid atherosclerosis

\begin{tabular}{|c|c|c|c|}
\hline Characteristic & No atherosclerosis & Atherosclerosis & p-value \\
\hline Subjects $n$ & 99 & 51 & \\
\hline Age yrs & $53.1 \pm 8.2$ & $57.5 \pm 5.9$ & 0.008 \\
\hline BMI $\mathbf{k g} \cdot \mathrm{m}^{-2}$ & $24.2 \pm 3.9$ & $24.7 \pm 3.7$ & 0.45 \\
\hline Hypertension & $35(35.4)$ & $24(47.1)$ & 0.17 \\
\hline \multicolumn{4}{|l|}{ Smoking status } \\
\hline Never smoker & $50(50.5)$ & $26(51.0)$ & 0.18 \\
\hline Former smoker & $29(29.3)$ & 20 (39.2) & \\
\hline Current smoker & $20(20.2)$ & $5(9.8)$ & \\
\hline Duration of asthma yrs & $17.9 \pm 13.2$ & $18.4 \pm 15.1$ & 0.81 \\
\hline
\end{tabular}

Data are presented as mean \pm SD or $n(\%)$, unless otherwise stated. For chlorofluorocarbon beclomethasone dipropionate, conversion factors are 1.25 for budesonide and 2.0 for fluticasone propionate, hydrofluoroalkane beclomethasone dipropionate and hydrofluoroalkane ciclesonide. BMI: body mass index; ICS: inhaled corticosteroids; FVC: forced vital capacity expressed; \% pred: \% predicted; FEV1: forced expiratory volume in $1 \mathrm{~s}$. ${ }^{*}$ : calculated from the cumulative dose of ICS administered during the preceding 2 yrs. p-values were obtained by t-test and Chi-squared test for comparisons between the two groups.

In an attempt to determine the anti-atherogenic properties of corticosteroids, we aimed to study asthmatic patients regularly treated with ICS. Asthma itself would not influence vascular inflammation since inflammation is chiefly restricted to airways in this disorder. In addition, ICS exert little metabolic effect. We therefore considered ICS-treated asthmatic patients suitable for a study to clarify the effects of corticosteroids on atherosclerosis.
To avoid the metabolic effects of corticosteroids that would potentially have a direct effect on atherosclerosis, we excluded patients who had been treated with regular oral and/or intravenous corticosteroids. Therefore, the atherosclerotic risk of the asthmatic patients was not high compared with the control subjects. Asthmatic patients were found to have significantly lower carotid IMT than control subjects in the study.

TABLE 3 Simple logistic regression analysis and stepwise multiple logistic regression analysis of independent factors for atherosclerosis of carotid arteries in asthmatic patients

\begin{tabular}{|c|c|c|c|c|}
\hline & \multicolumn{2}{|c|}{ Simple logistic regression analysis } & \multicolumn{2}{|c|}{ Stepwise multiple logistic regression analysi } \\
\hline & OR $(95 \% \mathrm{Cl})$ & $\mathrm{p}$-value & OR $(95 \% \mathrm{Cl})$ & $\mathrm{p}$-value \\
\hline Age per $1 \mathrm{yr}$ & $1.07(1.02-1.13)$ & 0.026 & $1.11(1.05-1.18)$ & 0.001 \\
\hline Dyslipidaemia & $2.88(1.42-6.05)$ & 0.004 & $2.58(1.19-5.75)$ & 0.018 \\
\hline Mean daily dose of ICS\# & $0.87(0.77-0.98)$ & 0.023 & $0.86(0.74-0.97)$ & 0.021 \\
\hline Diabetes mellitus & $2.09(0.68-6.47)$ & 0.19 & Not entered & \\
\hline Smoking status & $0.82(0.51-1.29)$ & 0.40 & Not entered & \\
\hline BMI per $1 \mathrm{~kg} \cdot \mathrm{m}^{-2}$ & $1.04(0.95-1.13)$ & 0.44 & Not entered & \\
\hline $\mathrm{FEV}_{1} / \mathrm{FVC} \%$ & $0.99(0.96-1.03)$ & 0.76 & Not entered & \\
\hline Duration of asthma per yr & $1.00(0.98-1.03)$ & 0.81 & Not entered & \\
\hline FVC $\%$ pred & $0.99(0.98-1.02)$ & 0.76 & Not entered & \\
\hline
\end{tabular}

Dyslipidaemia was: $0=$ absent; $1=$ present. Smoking status was: $0=$ never smoker; $1=$ former smoker; $2=$ current smoker. Diabetes mellitus was: $0=$ absent, $1=$ present. Hypertension was $0=a b s e n t ; 1=$ present. The dose of inhaled corticosteroids (ICS) is expressed as equipotent dose, with chlorofluorocarbon beclomethasone dipropionate as reference. For chlorofluorocarbon beclomethasone dipropionate, conversion factors are 1.25 for budesonide and 2.0 for fluticasone propionate, hydrofluoroalkane beclomethasone dipropionate and hydrofluoroalkane ciclesonide. BMI: body mass index; FEV1: forced expiratory volume in $1 \mathrm{~s}$; FVC: forced vital capacity; \% pred: \% predicted. \#: for the preceding 2 yrs per $100 \mu \mathrm{g} \cdot$ day $^{-1}$. 
Only a few studies have been carried out to analyse the effects of corticosteroids on the arteries as well as on cardiovascular events. Oral corticosteroid treatment has been reported to be associated with the progression of atherosclerosis, independent of traditional risk factors in patients with rheumatoid arthritis and systemic lupus erythematosus $[25,26]$. The large cohort study of VARAS-LORENZO et al. [9] reported that the use of oral corticosteroids was associated with a greater risk of acute myocardial infarction in $>40,000$ subjects from the general UK population. These studies suggest that oral corticosteroid treatment is associated with the progression of atherosclerosis. With regard to ICS, VARAS-LORENZO et al. [9] found that there was no increase in the risk of acute myocardial infarction. Another study also found that ICS may reduce the risk of acute myocardial infarction in asthmatic patients [13]. These reports suggest that ICS but not oral corticosteroids may have a role in preventing atherosclerosis.

The prevalence of carotid plaques in asthmatic patients was lower than in the control subjects $(p=0.06)$. Carotid IMT and carotid plaque are known to reflect biologically and genetically different aspects of the atherosclerotic process [27]. 21 asthmatic patients had a mean IMT of $\geqslant 1.1 \mathrm{~mm}, 24$ had a plaque lesion, and six had both phenotypes. We conclude that a mean IMT $\geqslant 1.1 \mathrm{~mm}$ and/or the presence of a plaque lesion provide the most sensitive definition of carotid atherosclerosis to predict cardiovascular disease. Our results suggest that both lesions on the carotid artery are repressed by chronic ICS administration in asthmatic patients.

The comparison between asthmatic patients with and without carotid atherosclerosis showed no significant difference in the prevalence of the traditional atherosclerosis risk factors of obesity, hypertension, diabetes mellitus and smoking status. The prevalence of hypertension was higher in patients with carotid atherosclerosis than in those without it, but the difference was not statistically significant. BMI and smoking did not contribute to atherosclerosis in this study. With regard to smoking, current smokers needed a larger daily dose of ICS to control asthma than former and never smokers [28]. The increase in the daily dose of ICS in smokers might be enough to protect against the progression of carotid atherosclerosis because the extent of inflammation was moderate. Most importantly, the mean daily dose of ICS for the preceding 2 yrs was significantly lower for patients with atherosclerosis. The influence of increasing the daily dose of ICS on a reduction in carotid atherosclerosis was proved by logistic regression analyses. The sample size of 150 was enough to have a power of $82 \%$ to detect an association between ICS and carotid atherosclerosis at a two-sided $\alpha$ of 0.05 , using the normal approximation formulae for logistic regression given by $\mathrm{HsIEH}$ [29]. Taken together, these results suggest that ICS treatment may have a dose-dependent anti-atherogenic effect in asthmatic patients.

In an animal experiment, corticosteroid administration was shown to reduce the rate of plaque initiation or progression despite an increase in circulating cholesterol and triglyceride levels [30]. Besides the direct inhibitory effects of corticosteroids on the expression of vascular adhesion molecules [10, 11], they also show acute cardiovascular effects through nontranscriptional activation of endothelial nitric oxide synthase
[31]. Relatively small amounts of ICS are absorbed into the circulation, which sometimes results in hypothalamic-pituitary-adrenal axis suppression, abnormal bone metabolism and cataracts $[4,5]$. There is no information about the effects of ICS on the cells that make up vessel walls. However, ICS have been shown to have a higher affinity for glucocorticoid receptor [4] and stronger anti-inflammatory effects on bronchial epithelial cells [32] than dexamethasone and triamcinolone acetonide. Recently, ICS use has been shown to be associated with lower cardiovascular mortality in females with asthma [33] Although atherosclerosis was not evaluated in that study, taken with our results, the lower cardiovascular mortality may partly be due to the anti-atherogenic effects of ICS.

One limitation of this study is that it was a case-control study and the sample size did not provide sufficient statistical power to elucidate the atheroprotective effects of ICS. Although further prospective studies in large number of patients are needed to confirm our findings, the results of this study indicate the potential of ICS for the prevention and treatment of some aspects of atherosclerosis.

In conclusion, many studies have reported unfavourable systemic effects of ISC, although they are the mainstay of long-term asthma treatment [3-5]. In contrast, we were able to show that carotid atherosclerosis was less severe in asthmatic patients treated with ICS than in control subjects. This favourable effect of ICS treatment was dose-dependent. This is the first study to report that ICS may have protective effects against atherosclerosis.

\section{SUPPORT STATEMENT}

This work was supported in part by grants from the Ministry of Education, Culture, Sports, Science and Technology of Japan and the Ministry of Health, Labour and Welfare of Japan (Soji Kasayama).

\section{STATEMENT OF INTEREST}

None declared.

\section{REFERENCES}

1 Expert Panel Report 3 (EPR-3): Guidelines for the diagnosis and management of asthma: Summary Report 2007. J Allergy Clin Immunol 2007; 120: S94-S138.

2 Statement by the British Thoracic Society, the British Paediatric Association, the Research Unit of the Royal College of Physicians of London, the King's Fund Centre, the National Asthma Campaign, the Royal College of General Practitioners, the General Practitioners in Asthma Group, the British Association of Accident and Emergency Medicine and the British Paediatric Respiratory Group. Guidelines on the management of asthma. Thorax 1993; 48: S1-S24.

3 Barnes PJ, Pedersen S. Efficacy and safety of inhaled corticosteroids in asthma. Report of a workshop held in Eze, France, October 1992. Am Rev Respir Dis 1993; 148: S1-S26.

4 Dahl R. Systemic side effects of inhaled corticosteroids in patients with asthma. Respir Med 2006; 100: 1307-1317.

5 Fujita K, Kasayama S, Hashimoto J, et al. Inhaled corticosteroids reduce bone mineral density in early postmenopausal but not premenopausal asthmatic women. J Bone Miner Res 2001; 16: 782-787.

6 Allen DB, Bielory L, Derendorf H, et al. Inhaled corticosteroids: past lessons and future issues. J Allergy Clin Immunol 2003; 112: S1-S40. 
7 Ross R. Atherosclerosis - an inflammatory disease. N Engl J Med 1999; 340: 115-126.

8 Nashel DJ. Is atherosclerosis a complication of long-term corticosteroid treatment? Am J Med 1986; 80: 925-929.

9 Varas-Lorenzo C, Rodriguez LA, Maguire A, et al. Use of oral corticosteroids and the risk of acute myocardial infarction. Atherosclerosis 2007; 192: 376-383.

10 Cronstein BN, Kimmel SC, Levin RI, et al. A mechanism for the antiinflammatory effects of corticosteroids: the glucocorticoid receptor regulates leukocyte adhesion to endothelial cells and expression of endothelial-leukocyte adhesion molecule 1 and intercellular adhesion molecule 1. Proc Natl Acad Sci USA 1992; 89: 9991-9995.

11 Simoncini $T$, Maffei S, Basta G, et al. Estrogens and glucocorticoids inhibit endothelial vascular cell adhesion molecule-1 expression by different transcriptional mechanisms. Circ Res 2000; 87: 19-25.

12 Bevilacqua MP, Nelson RM, Mannori G, et al. Endothelialleukocyte adhesion molecules in human disease. Annu Rev Med 1994; 45: 361-378.

13 Suissa $\mathrm{S}$, Assimes T, Brassard $\mathrm{P}$, et al. Inhaled corticosteroid use in asthma and the prevention of myocardial infarction. Am J Med 2003; 115: 377-381.

14 O'Leary DH, Polak JF, Kronmal RA, et al. Carotid-artery intima and media thickness as a risk factor for myocardial infarction and stroke in older adults. Cardiovascular Health Study Collaborative Research Group. N Engl J Med 1999; 340: 14-22.

15 Standards for the diagnosis and care of patients with chronic obstructive pulmonary disease (COPD) and asthma. This official statement of the American Thoracic Society was adopted by the ATS Board of Directors, November 1986. Am Rev Respir Dis 1987; 136: $225-244$.

16 Report of the Expert Committee on the Diagnosis and Classification of Diabetes Mellitus. Diabetes Care 1997; 20: 1183-1197.

17 Adams N, Bestall JM, Lasserson TJ, et al. Inhaled fluticasone versus inhaled beclomethasone or inhaled budesonide for chronic asthma in adults and children. Cochrane Database Syst Rev 2005; 2: CD002310.

18 Buhl R, Vinkler I, Magyar P, et al. Comparable efficacy of ciclesonide once daily versus fluticasone propionate twice daily in asthma. Pulm Pharmacol Ther 2006; 19: 404-412.

19 Lasserson TJ, Cates CJ, Jones AB, et al. Fluticasone versus HFAbeclomethasone dipropionate for chronic asthma in adults and children. Cochrane Database Syst Rev 2005; 4: CD005309.
20 Yamasaki Y, Kawamori R, Matsushima H, et al. Atherosclerosis in carotid artery of young IDDM patients monitored by ultrasound high-resolution B-mode imaging. Diabetes 1994; 43: 634-639.

21 Otsuki M, Hashimoto K, Morimoto Y, et al. Circulating vascular cell adhesion molecule-1 (VCAM-1) in atherosclerotic NIDDM patients. Diabetes 1997; 46: 2096-2101.

22 Salonen R, Seppanen K, Rauramaa R, et al. Prevalence of carotid atherosclerosis and serum cholesterol levels in eastern Finland. Arteriosclerosis 1988; 8: 788-792.

23 Akaike H. Information Theory and an Extension of the Maximum Likelihood Principle. Budapest, Academia Kaido, 1972.

24 Moreland LW, O'Dell JR. Glucocorticoids and rheumatoid arthritis: back to the future? Arthritis Rheum 2002; 46: 2553-2563.

25 del Rincon I, O'Leary DH, Haas RW, et al. Effect of glucocorticoids on the arteries in rheumatoid arthritis. Arthritis Rheum 2004; 50: 3813-3822.

26 Zeller CB, Appenzeller S. Cardiovascular disease in systemic lupus erythematosus: the role of traditional and lupus related risk factors. Curr Cardiol Rev 2008; 4: 116-122.

27 Spence JD. Measurement of intima-media thickness vs. carotid plaque: uses in patient care, genetic research and evaluation of new therapies. Int J Stroke 2006; 1: 216-221.

28 Thomson NC, Spears M. The influence of smoking on the treatment response in patients with asthma. Curr Opin Allergy Clin Immunol 2005; 5: 57-63.

29 Hsieh FY. Sample size tables for logistic regression. Stat Med 1989; 8: 795-802.

30 Makheja AN, Bloom S, Muesing R, et al. Anti-inflammatory drugs in experimental atherosclerosis. 7. Spontaneous atherosclerosis in WHHL rabbits and inhibition by cortisone acetate. Atherosclerosis 1989; 76: 155-161.

31 Hafezi-Moghadam A, Simoncini T, Yang Z, et al. Acute cardiovascular protective effects of corticosteroids are mediated by nontranscriptional activation of endothelial nitric oxide synthase. Nat Med 2002; 8: 473-479.

32 Atsuta J, Plitt J, Bochner BS, et al. Inhibition of VCAM-1 expression in human bronchial epithelial cells by glucocorticoids. Am J Respir Cell Mol Biol 1999; 20: 643-650.

33 Camargo CA Jr, Barr RG, Chen R, et al. Prospective study of inhaled corticosteroid use, cardiovascular mortality, and all-cause mortality in asthmatic women. Chest 2008; 134: 546-551. 